Огляди літератури, оригінальні дослідження, погляд на проблему, випадок з практики, короткі повідомлення УДК 616-056.52-085-06:616.728.3-018.3-007.24

DOI 10.11603/1811-2471.2020.v.i1.11085

\title{
ВЛИЯНИЕ МЕДИКАМЕНТОЗНОЙ ТЕРАПИИ ОЖИРЕНИЯ НА КЛИНИЧЕСКИЕ ПРОЯВЛЕНИЯ ОСТЕОАРТРОЗА КОЛЕННЫХ СУСТАВОВ НА ПЕРВИЧНОМ ЗВЕНЕ ЗДРАВООХРАНЕНИЯ
}

\author{
๑С. Б. Холбаев, Ш. А. Юсупов, Н. Э. Юлдашова, Н. Э. Сулайманова \\ Самаркандский государственный медицинский институт, Узбекистан
}

РЕЗЮМЕ. В статье рассмотрено действие терапии орлистатом на клинические проявления остеоартроза (ОА) коленных суставов у пациенток, страдающих ожирением на участке врача общей практике.

Цель - оценка влияния снижения массы тела на фоне приема орлистата на клинические проявления ОА КС у женщин с избыточной массой тела.

Материал и методы. В исследовании участвовало 5 врачей общей практики, и 50 их пациенток с симптоматическим ОА, соответствующим критериям Американской коллегии ревматологов (ACR).

Для определения эффективности проводимой терапии использовался опросник WOMАC. Оценку проводили по ВАШ в миллиметрах. Качество жизни (состояние здоровья пациента) определялось по ВАШ по опроснику EQ-5D. У пациентов оценивались неблагоприятные реакции за период наблюдения. Обработка данных проводилась с помощью методов описательной статистики.

Результаты. Терапия ожирения орлистатом позволила большинству пациентов с избыточной массой тела добиться ее клинически значимого снижения ( $\geq 5$ \%). Уменьшение массы тела при приеме специальных препаратов у пациентов с ожирением и ОА КС сопровождается уменьшением боли и улучшением функционального состояния КС, что благоприятно влияет на показатели качества жизни пациентов.

Выводы. Снижение массы тела, у пациентов с повышенным индексом массы тела и ОА коленных суставов, особенно при приеме препаратов, способствующих ее уменьшению, ведет к регрессу клинических проявлений гонартроза: уменьшению боли и улучшению функционального состояния. В связи с этим препараты, способствующие снижению массы тела, необходимо включать в схему лечения пациентов с ОА и ожирением.

КЛЮЧЕВЫЕ СЛОВА: остеоартроз; ожирение; орлистат.

Вступление. Эксперты Всемирной организации здравоохранения (ВОЗ) признали ожирение новой хронической «эпидемией» нашего времени. По последним оценкам ВОЗ, более миллиарда человек в мире имеют избыточную массу тела [1].

Известно, что ожирение часто предшествует развитию остеоартроза (ОА) коленных суставов (КС) и увеличивает риск прогрессирования ОА $[2,3]$. Риск развития ОА КС у пациентов с ожирением в 8 раз выше, чем у пациентов с нормальным индексом массы тела (ИМТ) [4]. Помимо этого, ожирение в значительной степени связано с прогрессированием рентгенологических изменений суставов в виде сужения суставной щели и увеличения размеров остеофитов [5]. По данным M. A. Cimmino и соавт. [6], существует взаимосВязь ожирения и интенсивности боли при ОА КС. Боль оценивалась по визуальной аналоговой шкале (ВАШ) и была максимальной у пациентов с ОА КС и ожирением. При коксартрозе и ОА суставов кистей в сочетании с ожирением боль была менее выраженной. R. Marks было установлено, что у пациентов с ОА КС и ожирением отмечалась большая интенсивность боли, чем у лиц с низким ИМТ $(p<0,05)[7]$.

При наблюдении 250 больных ОА КС в течение 2 лет отмечено увеличение массы тела более чем на $5 \%$, что сопровождалось увеличением боли в среднем на 27,1 мм, скованности на 18,4 мм и функциональной недостаточности на 99,3 мм по визуально-аналоговой шкале (ВАШ) [8].

Следовательно, в лечении ОА большое внимание должно уделяться коррекции избыточной массы тела. Систематический обзор литературы по более чем 50 консервативным методам лечения ОА показал значимость лечения ожирения при ОА и замедление прогрессирования заболевания при снижении массы тела [9].

Выделяют следующие виды лечения ожирения: соблюдение гипокалорийной диеты, физические упражнения, фармакологические и хирургические методы.

Национальный институт здоровья США (NIH) опубликовал клинические рекомендации по идентификации, оценке и лечению избыточной массы тела у взрослых. Основной принцип этих рекомендаций: «Первоначальная цель в лечении ожирения должна заключаться в уменьшении массы тела на 10 \% по сравнению с исходной» [10]. В исследовании, проведенном в США, показано, что существует зависимость выраженности клинических проявлений от степени потери массы тела. Показано, что снижение массы тела более чем на $10 \%$ достоверно улучшает показатели WOMAC по сравнению с потерей массы тела на 5 \% и менее. У 
Огляди літератури, оригінальні дослідження, погляд на проблему, випадок з практики, короткі повідомлення

пациентов с увеличением массы тела эти показатели стали хуже, чем были исходно [11].

Установлено, что при соблюдении диеты и выполнении физических упражнений удается достичь снижения массы тела только на 5 \% и лишь непродолжительно, сохранить полученный результат в течение длительного времени не удается большинству людей [12].

Добавление медикаментозной терапии к гипокалорийной диете не только облегчает потерю массы тела, но и может предотвратить ее повторное увеличение, которое наблюдается при использовании только гипокалорийной диеты. Согласно Европейским [13] и Российским [14] клиническим рекомендациям по лечению ожирения, назначение медикаментозных препаратов целесообразно тем пациентам с ожирением (ИМТ $\left.>30 \mathrm{kr} / \mathrm{M}^{2}\right)$, которые не могут достичь или удержать клинически значимую степень снижения массы тела при использовании диеты и физических нагрузок, включая пациентов с факторами риска развития сахарного диабета (СД) 2-го типа. Медикаментозная терапия показана пациентам с ИМТ >27 кг/м² и висцеральным типом ожирения при наличии заболеваний, связанных с ожирением $[13,14]$. Среди препаратов, зарегистрированных в Российской Федерации, для лечения ожирения в указанных группах больных рекомендуется орлистат [14]. Механизм его действия основан на подавлении активности желудочно-кишечных липаз, что приводит к уменьшению всасывания жира в кишечнике и, в сочетании с гипокалорийной диетой, вызывает значимое снижение массы тела.

Исследования показывают, что орлистат в комбинации с умеренно гипокалорийной диетой значительно уменьшает массу тела и ее повторную прибавку, улучшает течение сопутствующих ожирению заболеваний и повышает качество жизни, по сравнению с применением только диетотерапии. Эффективность орлистата при ожирении показана в большом числе рандомизированных плацебо-контролируемых исследований $[15,16]$.

Цель - оценка влияния снижения массы тела на фоне приема орлистата на клинические проявления ОА КС у женщин с избыточной массой тела на участке врача общей практики.

Материал и методы исследования. В исследовании участвовало 5 врачей общей практики, каждый из которых набирал последовательно 10 пациентов с симптоматическим ОА, соответствующим критериям Американской коллегии ревматологов (ACR) [17]. В открытое сравнительное рандомизированное шестимесячное исследование были включены 50 женщин в возрасте 4565 лет с ОА КС II или III стадии по Kellgren и ожирением. Интенсивность боли в КС составляла не менее 40 мм по ВАШ. Всеми пациентами было подписано информированное согласие на участие в исследовании. Больные были рандомизированы на две группы. Пациенты 1-й группы (n=25) принимали орлистат в дозе 120 мг (1 капсула) 3 раза в сутки во время или после еды в течение 6 месяцев в комплексе с гипокалорийной диетой и физической активностью. Во 2-й группе ( $n=25)$ проводилась немедикаментозная терапия ожирения (гипокалорийная диета и физическая активность).

Критерии исключения: травма КС в период до 3 месяцев до начала исследования, внутрисуставное введение любых препаратов в течение 6 недель до начала исследования, клинические признаки синовита коленного сустава, вторичный гонартроз, противопоказания к приему орлистата (синдром мальабсорбции, холестаз, гиперчувствительность к препарату или его компонентам), СД 1-го и 2-го типов в стадии декомпенсации углеводного обмена. Во время ежемесячных визитов пациенты были осмотрены врачами общей практики. Проводилась оценка суставного статуса, антропометрических параметров: роста, массы тела, ИМТ по формуле Кеттле (кг/м²), окружности талии и бедер. Каждый пациент был проконсультирован врачом общей практики, с которым обсуждались вопросы рационального питания и диетотерапии. Все пациенты находились на гипокалорийной диете с дефицитом 500-600 ккал по сравнению с расчетным показателем, с содержанием жиров <30\%, углеводов - 50-55 \%, белков - 15-20 \%. Каждый пациент на протяжении 6 месяцев заполнял дневник питания, в котором ежедневно указывал рацион питания, калорийность, количество жира в граммах. Во время каждого визита анализировались данные этих дневников. Всем пациентам были даны разъяснения по изменению образа жизни. На фоне диетотерапии женщинам рекомендовали проводить мероприятия, направленные на повышение двигательной активности. Были даны рекомендации по выполнению изометрических упражнений, которые способствуют повышению выносливости и силы мышц. Отработан комплекс упражнений, направленный на укрепление четырехглавой мышцы бедра (например, поднять выпрямленную ногу на 15 см в положении лежа на спине с притянутым мыском и удерживать ее несколько секунд), на сгибание коленного сустава в течение 15-20 мин ежедневно. Также были рекомендованы аэробные нагрузки (например, ходьба по ровной местности).

Для определения эффективности проводимой терапии использовался опросник WOMAC (Western Ontario and McMaster University) - для самостоятельной оценки больным выраженности 
Огляди літератури, оригінальні дослідження, погляд на проблему, випадок з практики, короткі повідомлення боли (в покое и при ходьбе - 5 вопросов), скованности (длительность и выраженность - 2 вопроса) и функциональной недостаточности в повседневной деятельности (17 вопросов). Оценку проводили по ВАШ в миллиметрах - от 0 (нет симптомов/ ограничений) до 100 (максимальная выраженность симптомов/ограничений). Качество жизни (состояние здоровья пациента) определялось по ВАШ от 0 до 100 мм по опроснику EQ-5D, где числом 100 обозначено наилучшее состояние здоровья, которое можно себе представить, а наихудшее состояние здоровья обозначено как 0. У пациентов оценивались неблагоприятные реакции за период наблюдения.

Статистический анализ проводился с помощью пакета прикладных программ Statistica 6.0. Обработка данных проводилась с помощью методов описательной статистики, для сравнения групп использовался критерий Стьюдента. Статистически значимыми считались различия при р<0,05.

Результаты и обсуждения. Пациенты обеих групп были сопоставимы по основным клиническим параметрам. Средний возраст включенных в исследование женщин составил $(55,9 \pm 5,8)$ года. Большинство женщин с ожирением в обеих группах имели II стадию гонартроза по KellgrenLawrence. Длительность OA в обеих группах в среднем составила 7,4 года (табл. 1).

Таблица 1. Клиническая характеристика пациенток с ожирением до назначения лечения

\begin{tabular}{|c|c|c|c|}
\hline Параметры & 1-я группа (n=25) & 2-я группа (n=25) & $P$ \\
\hline Средний возраст, годы, $\mathrm{M \pm \sigma}$ & $55,08 \pm 6,58$ & $57,92 \pm 4,77$ & 0,251 \\
\hline 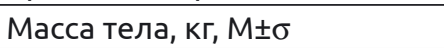 & $103,76 \pm 11,37$ & $102,18 \pm 17,4$ & 0,806 \\
\hline ИМТ, $\mathrm{K} г \mathrm{M}^{2}, \mathrm{M} \pm \sigma$ & $40,36 \pm 4,84$ & $38,77 \pm 5,62$ & 0,483 \\
\hline $\begin{array}{l}\text { Стадия гонартроза } \\
\text { по Kellgren-Lawrence, n (\%) } \\
\text { II } \\
\text { III }\end{array}$ & $\begin{array}{c}23(92) \\
2(8)\end{array}$ & $\begin{array}{l}22(88) \\
3(12)\end{array}$ & \\
\hline 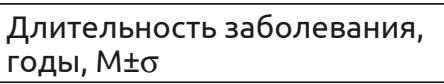 & $7,4 \pm 4,15$ & $7,4 \pm 3,77$ & 1,000 \\
\hline 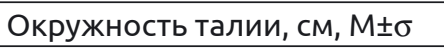 & $110,56 \pm 7,67$ & $111,32 \pm 10,64$ & 0,703 \\
\hline 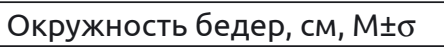 & $128,68 \pm 10,33$ & $127,38 \pm 15,65$ & 1,000 \\
\hline \multicolumn{4}{|l|}{ WOMAC, $M M, M \pm \sigma:$} \\
\hline Боль & $225,72 \pm 96,42$ & $230,08 \pm 74,14$ & 0,870 \\
\hline Скованность & $80,36 \pm 53,03$ & $108,52 \pm 41,91$ & 0,044 \\
\hline $\begin{array}{l}\text { Функциональная } \\
\text { недостаточность }\end{array}$ & $806,88 \pm 322,14$ & $886,72 \pm 354,35$ & 0,413 \\
\hline Суммарный & $1112,96 \pm 433,02$ & $1227,6 \pm 443,92$ & 0,363 \\
\hline $\begin{array}{l}\text { Качество жизни по ВАШ, мм, } \\
\text { М } \pm 0,413\end{array}$ & $49,88 \pm 16,21$ & $42,8 \pm 10,41$ & 0,070394 \\
\hline
\end{tabular}

Значительная доля больных с ожирением и гонартрозом имели сопутствующие заболевания. Чаще всего регистрировалась артериальная гипертензия - у 22 (88 \%) женщин 1-й группы и у 21 (84 \%) женщины 2-й группы. У 2 женщин в 1-й и во 2-й группах диагностирован СД 2-го типа. В обеих группах лечение ОА проводилось по стандартной схеме. В 1-й группе 23 женщины регулярно получали нестероидные противовоспалительные препараты (НПВП), 12 - хондропротекторы. Во 2-й группе 25 больных регулярно принимали НПВП, 11 хондропротекторы. Завершили исследование все 50 больных. У двух пациенток, получавших орлистат, на фоне погрешностей в диете (прием пищи, насыщенной животными жирами) развились неблагоприятные реакции в виде однократного эпизода жидкого стула, которые не потребовали отмены препарата. Комплаентность пациентов определялась посредством подсчета возвращенных капсул препарата во время визитов и составила 100 \%. Снижение массы тела отмечено в обеих группах (рис. 1). За 12 недель в 1-й группе оно составило 6,2 \%, во 2-й - 1,6 \%. За 24 недели на фоне терапии орлистатом масса тела снизилась в среднем на 10,4 кг (10,0 \%). 11 (44 \%) пациентов потеряли не менее $5 \%$ от исходной массы тела, 14 (56\%) - более $10 \%$. Во 2-й группе масса тела снизилась в среднем на 1 кг (0,9%). В период наблюдения с 12-й по 24-ю неделю снижения массы тела не наблюдалось.

Динамика изменений антропометрических показателей представлена в таблице 2 .

В обеих группах больных отмечено улучшение функционального статуса WOMAC на фоне снижения массы тела. Достоверное уменьшение боли и скованности наблюдалось у пациентов 1-й группы. Через 12 недель у пациентов 1-й группы на фоне снижения массы тела на 6,2 \% отмечено достоверное $(p<0,05)$ уменьшение боли по 
Огляди літератури, оригінальні дослідження, погляд на проблему, випадок з практики, короткі повідомлення

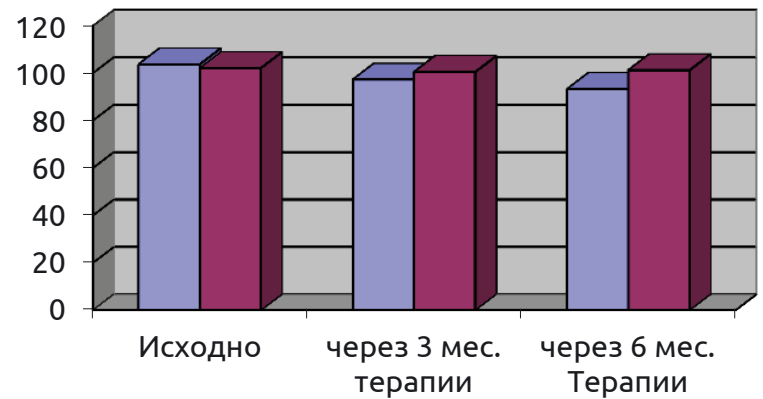

口Орлистат

口Без орлистата

Примечание. * - p<0,05 внутри групп, ** - p<0,05 между группами.

Рис. 1. Динамика снижения массы тела.

Таблица 2. Динамика изменений антропометрических показателей, $\mathrm{M \pm \sigma}$

\begin{tabular}{|l|c|c|c|c|c|c|}
\hline \multirow{2}{*}{ Показатель } & \multicolumn{2}{|c|}{ Исходно } & \multicolumn{2}{c|}{ Через 12 недель } & \multicolumn{2}{c|}{ Через 24 недели } \\
\cline { 2 - 7 } & 1 -я группа & 2-я группа & 1-я группа & 2-я группа & 1-я группа & 2 -я группа \\
\hline ОТ, см & $110,56 \pm 7,67$ & $111,32 \pm 10,64$ & $105,02 \pm 7,48$ & $108,52 \pm 8,43$ & $102,26 \pm 6,99 * *$ & $109,52 \pm 9,28$ \\
\hline ОБ, см & $128,68 \pm 10,33$ & $127,38 \pm 15,65$ & $122,6 \pm 10,56$ & $123,48 \pm 12,42$ & $118,5 \pm 9,77^{*}$ & $123,4 \pm 12,93$ \\
\hline
\end{tabular}

Примечание. ОТ - окружность талии, ОБ - окружность бедер. * - p<0,05 внутри каждой группы по сравнению с началом исследования; ** - $p<0,05$ между группами.

WOMAC на $40 \%$. Во 2-й группе, где масса тела снизилась на $1,6 \%$, интенсивность боли по WOMAC уменьшилась лишь на $15 \%$. Через 24 недли, на фоне терапии орлистатом, отмечено уменьшение боли по WOMAC на 52,6 \%. У пациентов 2-й группы этот показатель снизился на $28,8 \%$.

Через 24 недели у пациентов 1-й группы с потерей массы тела более чем на $10 \%$ отмечено достоверное уменьшение скованности и улуч- шение функционального состояния суставов, по сравнению с пациентами с потерей массы тела менее $5 \%$. Суммарный индекс WOMAC после 12 недель медикаментозной терапии ожирения у пациентов со снижением массы тела более чем на $5 \%$ снизился на $35,7 \%$, после 24 недель терапии - на 51,5 \% от исходных показателей. Во 2-й группе суммарный индекс WOMAC через 24 недели снизился на 19 \% (рис. 2).

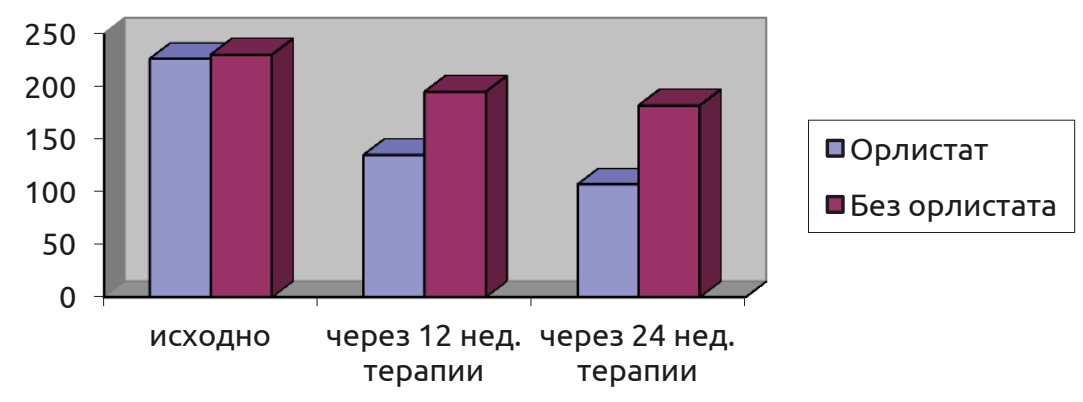

Pис. 2. Динамика боли по WOMAC.

Через 6 месяцев исследования качества жизни состояние здоровья по ВАШ, которое учитывалось как показатель, у пациентов 1-й группы улучшилось на $45 \%$, у пациентов 2 -й группы - на $23 \%(p<0,05)$. От приема НПВП отказались 20 (87 \%) больных 1-й группы и 6 (24 \%) пациентов 2-й группы.

Выводы. По результатам нашего исследования показано значимое снижение массы тела (на 10,0 \%) в группе больных ОА КС с ожирением на фоне приема орлистата.

Снижение массы тела способствует уменьшению интенсивности боли и улучшению функции
суставов.Уменьшение суммарного индекса WOMAC у пациентов с ожирением и ОА КС отмечалось уже после 3 месяцев терапии орлистатом. Эти изменения сохранялись в течение всего периода наблюдения.

Терапия ожирения орлистатом позволила большинству пациентов с избыточной массой тела добиться ее клинически значимого снижения ( $\geq 5 \%$ ). При этом $56 \%$ из них удалось снизить массу тела более чем на $10 \%$ от исходной.

Уменьшение массы тела при приеме специальных препаратов у пациентов с ожирением и 
Огляди літератури, оригінальні дослідження, погляд на проблему, випадок з практики, короткі повідомлення ОА КС сопровождается уменьшением боли и улучшением функционального состояния КС. Снижение массы тела и уменьшение клинических проявлений ОА КС благоприятно влияют на показатели

качества жизни пациентов, что позволяет рекомендовать включение в схему лечения пациентов с ОА и ожирением лекарственных средств, способствующих уменьшению массы тела.

\section{ЛИТЕРАТУРА}

1. Аметов А. С. Ожирение и современные методы лечения: Лекция для врачей / А. С. Аметов, Е. В. Доскина. - М. : РМАПО, 2011. - С. 3

2. Obesity, physically demanding work and traumatic knee injury are major risk factors for knee osteoarthritis a population-based study with a follow-up of 22 years / A. T. Toivanen, M. Heliövaara, O. Impivaara [et al.] // Rheumatology (Oxford). - 2010. - No. 49 (2). - P. 308-314. DOI: 10.1093/rheumatology/kep388

3. Incidence of severe knee and hip osteoarthritis in relation to different measures of body mass: a population-based prospective cohort study / L. S. Lohmander, M. Gerhardsson de Verdier, J. Rollof [et al.] // Ann. Rheum. Dis. - 2009. - No. 68 (4). - P. 490-496. DOI:10.1136/ard.2008.089748

4. Osteoarthritis: new insights. Part 1: The disease and its risk factors FREE / D. T. Felson, R. C. Lawrence, P. A. Dieppe [et al.] // Ann. Intern. Med. - 2000. - No. 133 (8). - P. 635646. DOI:10.7326/0003-4819-133-8-200010170-00016

5. Body mass index associated with onset and progression of osteoarthritis of the knee but not of the hip: The Rotterdam Study / M. Reijman, H. Pols, A. P. Bergink [et al.] // Ann. Rheum. Dis. - 2007. - No. 66. - P. 158-162. DOI: 10.1136/ard.2006.053538

6. Body mass and osteoarthritic pain: results from a study in general practice / M. A. Cimmino, R. Scarpa, R. Caporali [et al.] // Clin. Exp. Rheumatol. - 2013. - No. 31 (6). No. 843-P. 9.

7. Marks R. Obesity profiles with knee osteoarthritis: correlation with pain, disability, disease progression / R. Marks // Obesity (Silver Spring). - 2007. - No. 15 (7). P. 1867-74. DOI: 10.1038/oby.2007.221

8. Tanamas S.K., Wluka A.E. Association of weight gain with incident knee pain, stiffness, and functional dificulties: A longitudinal study / S. K. Tanamas, A. E. Wluka // Arthr. Care. Res. - 2013. - No. 65 (1). - P. 34-43. DOI: 10.1002/acr.21745

9. OARSI recommendations for the management of hip and knee osteoarthritis: part III: Changes in evidence following systematic cumulative update of research published through January 2009 / W. Zhang, G. Nuki, R.W. Mos-

\section{REFERENCES}

1. Ametov, A.S., \& Doskina, E.V. (2011). Ozhirenie $i$ sovremennye metody lecheniya: Lektsiya dlya vrachei [Obesity and modern methods of treatment: A lecture for doctors]. Moscow: RMAPO [in Russian].

2. Toivanen, A.T., Heliövaara, M., \& Impivaara, O. (2010). Obesity, physically demanding work and traumatic knee injury are major risk factors for knee osteoarthritis - a populationbased study with a follow-up of 22 years. Rheumatology (Oxford), 49 (2), 308-314. DOI: 10.1093/rheumatology/kep388.

kowitz [et al.] // Osteoarthr. Cartilage. - 2010. - No. 18 (4). P. 476-499. DOI: 10.1016/j.joca.2010.01.013

10. Evaluation, and Treatment of Overweight and Obesity in Adults / National Institutes of Health, National Heart Lung and Blood Institute: Clinical Guidelines on the Identification, 1998. - P. 98-483.

11. Riddle D. L. Body weight changes and corresponding changes in pain and function in persons with symptomatic knee osteoarthritis: A cohort study / D. L. Riddle, P. W. Stratford // Arthr Care Res. - 2013. - No. 65 (1). P. 15-22. DOI: $10.1002 /$ acr.21692

12. Effects of intensive diet and exercise on knee joint loads, inflammation, and clinical outcomes among overweight and obese adults with knee osteoarthritis: the IDEA randomized clinical trial / S. P. Messier, S. L. Mihalko, C. Legault [et al.] // JAMA. -2013. - No. 310 (12). - P. 12631273. DOI: $10.1001 /$ jama.2013.277669

13. Management of Obesity in Adults: European Clinical Practice Guidelines / C. Tsigosa, V. Hainerb, A. Basdevantc [et al.] // Obesity Facts. - 2008. - No. 1. - P. 106116. DOI: $10.1159 / 000126822$

14. Бондаренко И. З. Лечение морбидного ожирения. Национальные клинические рекомендации / И. З. Бондаренко, С. А. Бутрова, Н. П. Гончаров // Ожирение и метаболизм. - 2011. - № 3. - С. 75-83.

15. X-PERT: weight reduction with orlistat in obese subjects receiving a mildly or moderately reduced-energy diet. Early response to treatment predicts weight maintenance / H. Toplak, O. Ziegler, U. Keller [et al.] // Diabetes Obes Metab. - 2005. - No. 7. - P. 699-708. DOI: 10.1111/j.1463 1326.2005.00483.x

16. One-year treatment of obesity: a randomized, double-blind, placebo-controlled, multicenter study of orlistat, a gastrointestinal lipase inhibitor / N. Finer, W. P. T. James, P. G. Kopelman [et al.] // Int. J. Obes. Relat. Metab Disord. 2000. - No. 24. - P. 306-313. DOI: 10.1038/sj.ijo.0801128

17. The American College of Rheumatology criteria for the classification and reporting of osteoarthritis of the hip / R. Altman, G. Alarcon, D. Appelrouth [et al.] // Arthritis Rheum. - 1991. - No. 34. - P. 505-514. DOI: 10.1002/ art. 1780340502

3. Lohmander, L.S., Gerhardsson de Verdier, M., \& Rollof, J. (2009). Incidence of severe knee and hip osteoarthritis in relation to different measures of body mass: a population-based prospective cohort study. Ann. Rheum. Dis., 68 (4), 490-496. DOI: 10.1136/ard.2008.089748

4. Felson, D.T., Lawrence, R.C., \& Dieppe, P.A. (2000). Osteoarthritis: new insights. Part 1: The disease and its risk factors FREE. Ann. Intern. Med., 133 (8), 635-646. DOI:10.7326/0003-4819-133-8-200010170-00016 
Огляди літератури, оригінальні дослідження, погляд на проблему, випадок з практики, короткі повідомлення

5. Reijman, M., Pols, H., \& Bergink, A.P. (2007). Body mass index associated with onset and progression of osteoarthritis of the knee but not of the hip: The Rotterdam Study. Ann. Rheum. Dis., 66, 158-162. doi: 10.1136/ard.2006.053538.

6. Cimmino, M.A., Scarpa, R., \& Caporali, R. (2013). Body mass and osteoarthritic pain: results from a study in general practice. Clin. Exp. Rheumatol., 31 (6), 843-849.

7. Marks, R. (2007). Obesity profiles with knee osteoarthritis: correlation with pain, disability, disease progression. Obesity (Silver Spring), 15 (7), 1867-1874. DOI: 10.1038/oby.2007.221

8. Tanamas, S.K., \& Wluka, A.E. (2013). Association of weight gain with incident knee pain, stiffness, and functional dificulties: A longitudinal study. Arthr. Care Res., 65 (1), 34-43. DOI: $10.1002 /$ acr.21745

9. Zhang, W., Nuki, G., \& Moskowitz, R.W. (2010). OARSI recommendations for the management of hip and knee osteoarthritis: part III: Changes in evidence following systematic cumulative update of research published through January 2009. Osteoarthr. Cartilage, 18 (4), 476-499. DOI: 10.1016/j.joca.2010.01.013

10. (1998). National Institutes of Health, National Heart Lung and Blood Institute. Evaluation, and Treatment of Overweight and Obesity in Adults. Clinical Guidelines on the Identification.

11. Riddle, D.L., \& Stratford, P.W. (2013). Body weight changes and corresponding changes in pain and function in persons with symptomatic knee osteoarthritis: A cohort study. Arthr. Care Res., 65 (1), 15-22. DOI: 10.1002/ acr.21692
12. Messier, S.P., Mihalko, S.L., \& Legault, C. (2013). Effects of intensive diet and exercise on knee joint loads, inflammation, and clinical outcomes among overweight and obese adults with knee osteoarthritis: the IDEA randomized clinical trial. JAMA, 310(12),1263-1273. DOI: 10.1001/jama.2013.277669

13. Tsigosa, C., Hainerb, V., \& Basdevantc, A. (2008). Management of Obesity in Adults: European Clinical Practice Guidelines. Obesity Facts, 1, 106-116. DOI: 10.1159/000126822

14. Bondarenko, I.Z., Butrova, S.A., \& Goncharov, N.P. (2011). Lecheniye komorbidnogo ozhyreniye. Natsionalnye klinicheskiye rekomendatsii [Treatment of morbid obesity. National clinical guidelines]. Ozhirenie i metabolism - Obesity and Metabolism, 3, 75-83 [in Russian].

15. Toplak, H., Ziegler, O., \& Keller, U. (2005). X-PERT: weight reduction with orlistat in obese subjects receiving a mildly or moderately reduced-energy diet. Early response to treatment predicts weight maintenance. Diabetes Obes. Metab., 7, 699-708. DOI: 10.1111/j.1463 1326.2005.00483.x

16. Finer, N., James, W.P.T., \& Kopelman, P.G. (2000). One-year treatment of obesity: a randomized, doubleblind, placebo-controlled, multicenter study of orlistat, a gastrointestinal lipase inhibitor. Int. J. Obes. Relat. Metab. Disord., 24, 306-313. DOI: 10.1038/sj.ijo.0801128

17. Altman, R., Alarcon, G., \& Appelrouth, D. (1991). The American College of Rheumatology criteria for the classification and reporting of osteoarthritis of the hip. Arthritis Rheum., 34, 505-514. DOI: 10.1002/art.1780340502

\section{ВПЛИВ МЕДИКАМЕНТОЗНОЇ ТЕРАПІЇ ОЖИРІННЯ НА КЛІНІЧНІ ПРОЯВИ ОСТЕОАРТРОЗУ КОЛІННИХ СУГЛОБІВ НА ПЕРВИННІЙ ЛАНЦІ ОХОРОНИ ЗДОРОВ'Я} ๑С. Б. Холбаев, Ш. А. Юсупов, Н. Е. Юлдашова, Н. Е. Сулайманова

Самаркандський державний медичний інститут, узбекистан

РЕзЮМЕ. У статті розглянута дія терапії орлістатом на клінічні прояви остеоартрозу (ОА) колінних суглобів у пацієнток, які страждають на ожиріння, на ділянці лікаря загальної практики.

Мета - оцінка впливу зниження маси тіла на тлі прийому орлістату на клінічні прояви ОА КС у жінок з надмірною масою тіла.

Матеріал і методи. У дослідженні брали участь 5 лікарів загальної практики і 50 їхніх пацієнток із симптоматичним ОА, що відповідає критеріям Американської колегії ревматологів (ACR).

Для визначення ефективності проведеної терапії використовувався опитувальник WOMAC. Оцінку проводили за ВАШ у міліметрах. Якість життя (стан здоров'я пацієнта) визначали за BAШ за опитувальником EQ-5D. У пацієнтів оцінювали несприятливі реакції за період спостереження. Обробку даних проводили за допомогою методів описової статистики.

Результати. Терапія ожиріння орлістатом дозволила більшості пацієнтів з надлишковою масою тіла домогтися її клінічно значущого зниження ( $\geq 5$ \%). Зменшення маси тіла при прийомі спеціальних препаратів у пацієнтів з ожирінням і ОА КС супроводжується зменшенням болю і поліпшенням функціонального стану КС, що сприятливо впливає на показники якості життя пацієнтів.

Висновки. Зниження маси тіла у пацієнтів з підвищеним індексом маси тіла і ОА колінних суглобів, особливо при прийомі препаратів, що сприяють її зменшенню, веде до регресу клінічних проявів гонартрозу: зменшення болю і поліпшення функціонального стану. У зв'язку з цим препарати, що сприяють зниженню маси тіла, необхідно включати в схему лікування пацієнтів з ОА і ожирінням.

КЛючОВІ СЛОВА: остеоартроз; ожиріння; орлістат. 
Огляди літератури, оригінальні дослідження, погляд на проблему, випадок з практики, короткі повідомлення

\section{THE EFFECT OF DRUG THERAPY IN PATIENTS WITH OBESITY ON THE CLINICAL MANIFESTATIONS OF KNEE OSTEOARTHRITIS}

\section{@S. B. Kholbayev, Sh. A. Yusupov, N. E. Yuldashova, N. E. Sulaymanova} Samarkand State Medical Institute, Republic of Uzbekistan, Samarkand

SUMMARY. The effect of orlistat therapy on the clinical manifestations of osteoarthrosis (OA) of the knee joints in patients suffering from obesity at the site of a general practitioner is considered in the article.

The aim - assessment of the effect of weight loss on the background of orlistat administration on clinical manifestations of OA of knee joints in women with overweight.

Material and Methods. The study involved 5 general practitioners and 50 patients with symptomatic OA that met the criteria of the American College of Rheumatologists (ACR).

The WOMAC questionnaire was used to determine the effectiveness of the therapy. The evaluation was performed by VAS in millimeters. Quality of life (patient health) was determined by VAS questionnaire EQ-5D. Patients were evaluated for adverse reactions during the observation period. Data processing was performed using descriptive statistics methods.

Results. Orlistat obesity therapy has the potential to achieve clinically significant reductions ( $\geq 5 \%$ ). Weight loss when taking special drugs in patients with obesity and OA of the knee joints is accompanied by a decrease in pain and improvement in the functional state of knee joints, which has a positive effect on the quality of life of patients.

Conclusions. Weight loss, in patients with high body mass index and OA of the knee joints, especially when taking drugs that contribute to its reduction, leads to regression of clinical manifestations of gonarthrosis: reducing pain and improving functional status. In this regard, drugs that contribute to weight loss, should be included in the scheme of treatment of patients with $\mathrm{OA}$ and obesity.

KEY WORDS: osteoarthritis; overweight patients; orlistat. 